\title{
A Rare Case of Persistent Pneumothorax in Non-Small Cell Lung Cancer on Bevacizumab Therapy
}

Hidefumi Koh ${ }^{1 *}$, Nobufumi Kamiishi ${ }^{1}$, Yoshishige Kimura ${ }^{2}$, Atsushi Tajima ${ }^{2}$, Toshiaki Yagami ${ }^{3}$ and Makio Mukai ${ }^{4}$

${ }^{1}$ Division of Pulmonary Medicine, Department of Internal Medicine, Saiseikai Utsunomiya Hospital, Tochigi, Japan

${ }^{2}$ Department of General Thoracic Surgery, Saiseikai Utsunomiya Hospital, Tochigi, Japan

${ }^{3}$ Department of Radiology, Saiseikai Utsunomiya Hospital, Tochigi, Japan

${ }^{4}$ Department of Pathology, Keio University School of Medicine, Tokyo, Japan

\begin{abstract}
Bevacizumab which is a humanized murine monoclonal antibody against vascular endothelial growth factor, given in combination with carboplatin and paclitaxel, was granted a labeling extension treatment of advanced-stage Non-Small Cell Lung Cancer (NSCLC). Common toxic effects include hypertension, proteinuria, and neutropenia. Rare complications include bleeding, particularly hemoptysis, and surgical wound dehiscence, but pneumothorax in NSCLC is rare. We report a case of persistent pneumothorax related to bevacizumab in a patient with NSCLC.
\end{abstract}

Keywords: Non-small cell lung cancer; Bevacizumab; Pneumothorax

\section{Introduction}

Bevacizumab is used as a standard regimen for chemotherapy in patients with Non-small cell lung cancer (NSCLC) [1,2]. Common toxic effects include hypertension, proteinuria, and neutropenia. However, peumothorax occurring after Bevacizumab-containing chemotherapy in NSCLC has been rarely described [3].

\section{Case Report}

54-year-old man was admitted in March 2010 to our hospital because of dyspnea and right pleural effusion. He had smoked three packs of cigarettes per day for thirty four years. Chest X-ray showed right pleural effusion (Figure 1A). Chest CT disclosed right pleural effusion and bilateral diffuse Low Attenuation Area (LAA). To determine the cause of pleural effusion, thoracentesis was performed and showed exudative pleural effusion. Pleural fluid cytology was consistent with adenocarcinoma (Figure 1B). Epidermal Growth Factor Receptor mutation was negative. Diagnostic workup was performed, and finally he was diagnosed as advanced-stage NSCLC. To control malignant pleural effusion, chemical pleurodesis was performed. And then, he administered two cycles of Cisplatin (CDDP) with Pemetrexed as first-line chemotherapy. It was not effective so that second-line chemotherapy of Carboplatin (CBDCA), Paclitaxel (PTX) with Bevacizumab was administered and continued six cycles. Chest $\mathrm{CT}$ after second-line chemotherapy showed right pneumothorax with pleural
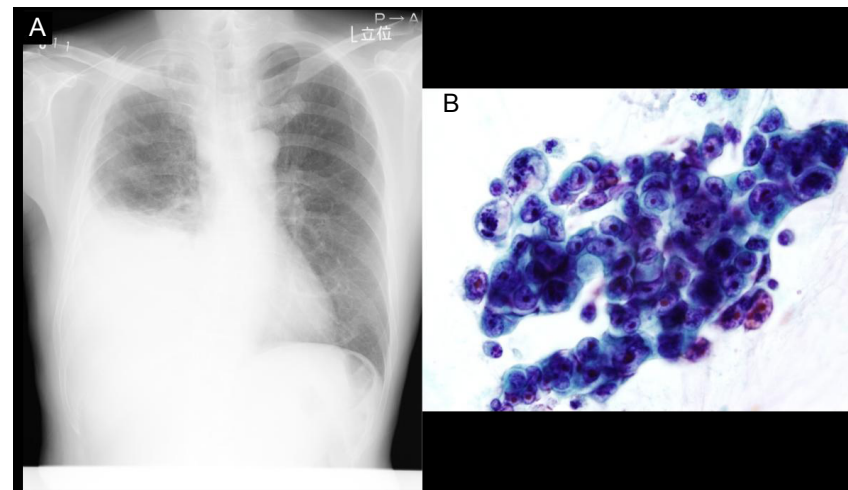

Figure 1: (A) Chest $X$-ray on admission demonstrates right pleural effusion

(B) Pleural fluid cytology: There are clusters of tumor cells with high nuclearcytoplasmic (N/C) ratio, granular chromatin and nucleoli (Papanicolaou stain; $\mathrm{x} 400)$

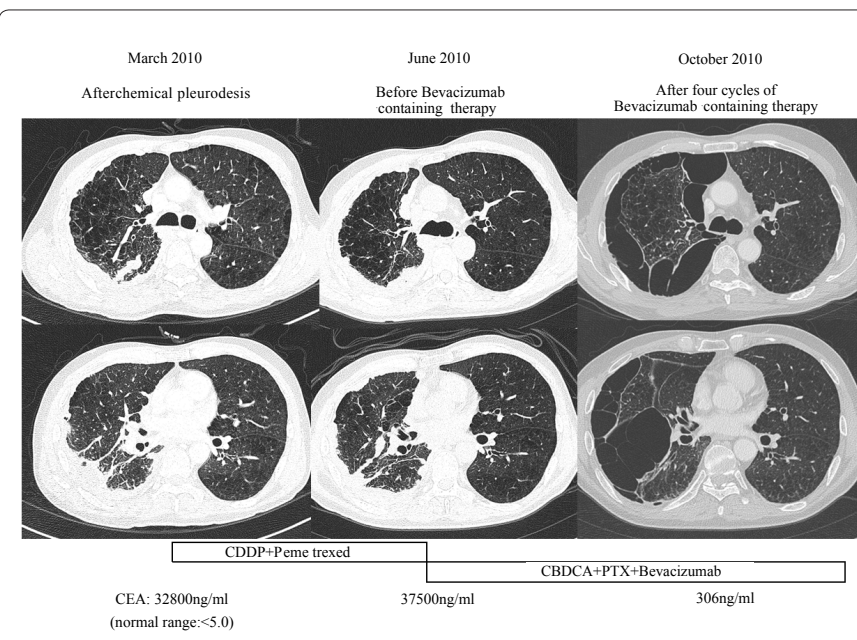

Figure 2: Chest CT showed right pneumothorax with pleural adhesion. Pneumothorax persisted throughout the clinical course.

adhesion (Figure 2). Nevertheless pneumothorax persisted throughout the clinical course; his condition was stable 28 months after diagnosis. As shown in Figure 2, pneumothorax with pleural adhesion persisted, but his respiratory failure did not worsened. Therefore, pneumothorax was not treated using chest tube or thoracoscopy. Carcinoembryonic Antigen (CEA) was gradually reduced after chemotherapy.

\section{Discussion}

Bevacizumab, which is a humanized monoclonal antibody against Vascular Endothelial Growth Factor (VEGF), is used as a standard regimen for chemotherapy in patients with NSCLC [1,2]. Common

*Corresponding author: Hidefumi Koh, Division of Pulmonary Medicine Department of Internal Medicine, Saiseikai Utsunomiya Hospital, 911-1 Takebayashimachi, Utsunomiya-shi, Tochigi 321-0974, Japan, Tel: +81-28-6265500; Fax: +81-28-626-5594; E-mail: hdfmkoh@yahoo.com

Received January 13, 2013; Accepted March 25, 2013; Published March 26 2013

Citation: Koh H, Kamiishi N, Kimura Y, Tajima A, Yagami T, et al. (2013) A Rare Case of Persistent Pneumothorax in Non-Small Cell Lung Cancer on Bevacizumab Therapy. J Pulmon Resp Med S14: 001. doi:10.4172/2161-105X.S14-001

Copyright: (c) $2013 \mathrm{Koh} \mathrm{H}$, et al. This is an open-access article distributed under the terms of the Creative Commons Attribution License, which permits unrestricted use, distribution, and reproduction in any medium, provided the original author and source are credited. 
toxic effects include hypertension, proteinuria, and neutropenia. Rare complications include bleeding, particularly hemoptysis, and surgical wound dehiscence. Pneumothorax related to bevacizumab has been rarely described [3]. To our knowledge, there are a few reports that peumothorax occurring after Bevacizumab-containing chemotherapy. Yang et al. [4] reported a case of peumothorax occurring after Bevacizumab-containing chemotherapy in colorectal cancer with lung metastases. They treated pneumothorax with small caliber chest tube and the tube was removed 5 days later. No recurrence was observed. They described that a small caliber chest tube was considered effective in management of uncomplicated peumotohrax. On the other hand, one patient died of recurrent bilateral pneumothoraces in phase II study of doxorubicin and bevacizumab for patients with metastatic soft tissue sarcomas [5]. Although pnemothorax resulting from treatment is a rare phenomenon [6], there was one case report of patients with lung metastases who developed recurrent pneumothoraces while receiving doxorubicin-based combination regimen [7]. Therefore, they speculated that recurrent pneumothoraces was treatment-related, but it was not clear whether doxorubicin or bevacizumab was main reason of pneumothoraces. Generally, several mechanisms of pneumothorax have been considered; Subpleural bulla rapture after chemotherapy, fistulas induced by tumor lysis due to chemotherapy, and air leak due to pulmonary infarction from tumor emboli. In our case, several reasons have been considered; first reason was rapture of bulla by diffuse LAA in both lungs, and second reason was subpleural bulla rapture and fistula formation after disappearing pleural dissemination by Bevacizumab-containing therapy. Furthermore VEGF is a strong player in lung structure maintenance $[8,9]$ and it has been reported chronic blockade of VEGF receptors causes alveolar septal cell apoptosis and lung capillary loss [10]. Hence Bevacizumab may cause pneumothorax in some patient; careful follow up will be needed.

\section{Conclusion}

Pneumothorax in patients with NSCLC after Bevacizumab- containing therapy is extremely rare. However, pneumothorax should not be overlooked because it is sometimes severe pulmonary complication, and should be considered in patients with NSCLC who have diffuse LAA or diagnosed as chronic obstructive pulmonary disease.

\section{References}

1. Sandler A, Gray R, Perry MC, Brahmer J, Schiller JH, et al. (2006) Paclitaxelcarboplatin alone or with bevacizumab for non-small-cell lung cancer. $\mathrm{N}$ Engl $\mathrm{J}$ Med 355: 2542-2550

2. Leighl NB, Zatloukal P, Mezger J, Ramlau R, Moore N, et al. (2010) Efficacy and safety of bevacizumab-based therapy in elderly patients with advanced or recurrent nonsquamous non-small cell lung cancer in the phase III BO17704 study (AVAiL). J Thorac Oncol 5: 1970-1976.

3. Chen HX, Cleck JN (2009) Adverse effects of anticancer agents that target the VEGF pathway. Nat Rev Clin Oncol 6: 465-477.

4. Yang SH, Lin JK, Chen WS, Lin TC, Yang SH, et al. (2011) Pneumothorax after bevacizumab-containing chemotherapy: a case report. Jpn J Clin Onco 41: 269-271.

5. D'Adamo DR, Anderson SE, Albritton K, Yamada J, Riedel E et al. (2005) Phase II study of doxorubicin and bevacizumab for patients with metastatic soft-tissue sarcomas. J Clin Oncol 23: 7135-7142.

6. Schulman P, Cheng E, Cvitkovic E, Golbey R (1979) Spontaneous pneumothorax as a result of intensive cytotoxic chemotherapy. Chest 75: 194 196.

7. Fenlon HM, Carney D, Breatnach E (1965) Case report: Bilateral recurrent tension pneumothorax complicating combination chemotherapy for soft tissue sarcoma. Clin Radiol 51: 302-304.

8. Voelkel NF, Cool CD (2003) Pulmonary vascular involvement in chronic obstructive pulmonary disease. Eur Respir J Suppl 46: 28s-32s.

9. Medford AR, Millar AB (2006) Vascular endothelial growth factor (VEGF) in acute lung injury (ALI) and acute respiratory distress syndrome (ARDS) paradox or paradigm? Thorax 61: 621-626.

10. Kasahara Y, Tuder RM, Taraseviciene-Stewart L, Le Cras TD, Abman S, et al. (2000) Inhibition of VEGF receptors causes lung cell apoptosis and emphysema. J Clin Invest 106: 1311-1319.
This article was originally published in a special issue, Pulmonology- Case Reportshandled by Editor(s). Dr. Anna Blumental-Perry, Memorial University Medical Center, USA. Dr. Bradley Allen Maron, Brigham and Women's Hospital, USA. 